\title{
Laboreal
}

Volume 17 №2 | 2021

Trabalho e Território

\section{Imperativos de los sistemas socio-técnicos y temporalidades humanas (ritmos biológicos y sociales, ciclos de vida e historia profesional)}

Imperativos dos sistemas sociotécnicos e temporalidades humanas (ritmos biológicos e sociais, ciclo de vida e história profissional) Impératifs des systèmes socio-techniques et temporalités humaines (rythmicités biologiques et sociales, cycle de vie et histoire professionnelle) Requirements of socio-technical systems and human temporalities (biological and social rhythms, life cycle and professional history)

\section{Charles Gadbois}

\section{OpenEdition}

\section{Journals}

Edición electrónica

URL: https://journals.openedition.org/laboreal/18134

DOI: $10.4000 /$ laboreal. 18134

ISSN: 1646-5237

Editor

Universidade do Porto

Referencia electrónica

Charles Gadbois, «Imperativos de los sistemas socio-técnicos y temporalidades humanas (ritmos biológicos y sociales, ciclos de vida e historia profesional)», Laboreal [En línea], Volume 17 №2 | 2021, Publicado el 01 diciembre 2021, consultado el 05 diciembre 2021. URL: http:// journals.openedition.org/laboreal/18134; DOI: https://doi.org/10.4000/laboreal.18134

Este documento fue generado automáticamente el 5 diciembre 2021

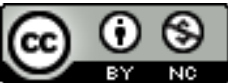

Laboreal está licenciado com uma Licença Creative Commons - Atribuição-NãoComercial 4.0 Internacional. 


\section{Imperativos de los sistemas socio- técnicos y temporalidades humanas (ritmos biológicos y sociales, ciclos de vida e historia profesional)}

Imperativos dos sistemas sociotécnicos e temporalidades humanas (ritmos

biológicos e sociais, ciclo de vida e história profissional)

Impératifs des systèmes socio-techniques et temporalités humaines (rythmicités

biologiques et sociales, cycle de vie et histoire professionnelle)

Requirements of socio-technical systems and human temporalities (biological and social rhythms, life cycle and professional history)

Charles Gadbois

REFERENCIA

Texto original:

Gadbois, Ch. (1993). Impératifs des systèmes socio-techniques et temporalités humaines (Rythmicités biologiques et sociales, cycle de vie et histoire professionnelle). In Actes $d u$ colloque de prospective soutenu par le PIR Cognisciences du CNRS: «Recherches pour l'ergonomie » (pp. 183-186). Université de Toulouse-le-Mirail.

NOTA DEL EDITOR

Traducido por

Gabriela Cuenca (gcuenca@frba.utn.edu.ar) 
1 Los imperativos tanto económicos como técnicos del funcionamiento de los sistemas socio-técnicos conducen cada vez más frecuentemente a imponer a los operadores un marco temporal de trabajo que no se corresponde con la vida común del ser humano. La práctica de la alternancia de turnos, según las formulas clásicas y emblemáticas del tipo $3 \times 8,4 \times 8$, tienden a aumentar, sobre todo ante el reciente cuestionamiento sobre la prohibición del trabajo nocturno para las mujeres. Además, en los últimos años han aparecido otras fórmulas (equipos de noche a tiempo parcial, equipos de fin de semana, 4 x 10, turnos de 12 horas) que plantean cuestiones de la misma índole.

2 Actualmente, estas cuestiones son mucho menos conocidas de lo que se podría pensar. Se encuentran en varias disciplinas que tienen bases y campos de investigación constituidos de forma desigual, siendo necesario desarrollar entre ellas articulaciones poco exploradas hasta ahora.

3 Las exigencias impuestas al ritmo biológico circadiano que sostiene a todo el funcionamiento del organismo humano representan un sector de investigación bien definido y muy relacionado con el campo de la investigación cronobiológica. Esto se manifiesta en la existencia de una red internacional organizada (coloquios periódicos, canales de publicación, etc.). Sin embargo, la mayoría de los trabajos en este campo se ven afectados por una limitación importante que es partir de un punto de vista implícito según el cual las variaciones de la actividad del operador serían el reflejo directo de las variaciones circadianas de sus capacidades. El enfoque ergonómico, basado en la observación directa in situ de la actividad real, lleva a constatar que este modelo es demasiado simplificado y que es necesario construir nuevos modelos que articulen el modelo ergonómico de la actividad laboral con el modelo cronobiológico de la eficiencia humana (Gadbois \& Queinnec, 1984; Gadbois, 1990).

Con este espíritu, en otoño de 1990 se creó el grupo "Crono-Ergonomía" del PIRTTEM (Programme Interdisciplinaire de Recherche sur le Travail, les Technologies, l'Emploi et les Modes de vie), que reunía a investigadores franceses cuyos trabajos abordaban la interacción de los ritmos circadianos en las actividades humanas, con diferentes enfoques (especialmente los que se desarrollan en el laboratorio y los realizados en terreno: ritmos de trabajo, ritmos escolares, etc.). Los intercambios y los inicios de la cooperación en el seno de este grupo, así como la organización del Coloquio Internacional "Shiftwork and job demands", celebrado en París en julio de 1991, atestiguan el interés de esta perspectiva. A pesar de la desaparición del PIRTTEM, los miembros del grupo desean continuar confrontando sus puntos de vista, sus conocimientos y aumentar sus intercambios; siendo la realización de los estudios de campo, uno de los medios preferidos para ello. Queda por ver cuáles son exactamente las condiciones precisas dichos estudios. Sin apoyo, tanto institucional como material, tal empresa, por muy justificada que esté en el plano intelectual, correría el riesgo de ser pospuesta a días más favorables.

5 Sin embargo, un mejor conocimiento del ritmo biológico circadiano no sería suficiente para abordar adecuadamente el diseño de los marcos temporales de trabajo. De hecho, los problemas que plantean estos marcos se originan en gran medida por su desajuste con los ritmos generales de la vida social (Gadbois, 1990b). Incluso es bien sabido que, en la práctica las consideraciones sociales son las que tienen importancia en las decisiones relacionadas con la organización del tiempo de trabajo. 
6 Sin embargo, por razones que deben ser examinadas, es evidente que las investigaciones siguen siendo insuficientes y que muy pocas se basan en un problema teórico bien elaborado. Hay una carencia a cubrir y es la de establecer mejores puentes con determinados sectores de la psicología social y la sociología, que desarrollan en otros campos, marcos teóricos y técnicas de análisis potencialmente pertinentes para abordar la organización del tiempo de trabajo.

7 Si bien superar los efectos de la constante compartimentación, no es la empresa más cómoda, es imprescindible, para abordar las interacciones entre la vida laboral y la no laboral. En este campo, sin embargo, durante la última década, se han desarrollado nuevos enfoques, cuya implementación en el tema del "tiempo de trabajo" debería ser fructífera.

8 Además del florecimiento de la literatura anglosajona en torno al tema "Coping with stress at work", el aporte más interesante a considerar aquí es sin duda el de la problemática del "sistema de actividades" de J. Curie y su equipo (Curie \& Hajjar, 1987). Lo que queda por hacer es crear las condiciones que favorezcan el desarrollo de esta perspectiva teórica en el campo de los marcos temporales del trabajo, y más allá, en las interacciones más amplias entre biólogos, psicólogos y sociólogos.

9 Aunque consideremos el ámbito de la actividad laboral o el de las relaciones entre vida de trabajo/vida fuera del trabajo, los problemas vinculados a los marcos temporales del trabajo solo pueden entenderse adecuadamente si se consideran desde un punto de vista diacrónico, a partir de la historia individual de los trabajadores afectados. Hoy, podemos dar por sentado la idea que los horarios atípicos de trabajo conducen al desarrollo - de forma interdependiente- de regulaciones individuales, inscritas en la historia personal (ciclo de vida) y profesional (carrera) de los trabajadores y políticas de gestión (explícitas o no) de las poblaciones de asalariados que trabajan en estos horarios de trabajo atípicos.

10 Este punto de vista procede en parte de la evaluación de las investigaciones anteriores sobre el trabajo por turnos y en particular de la reflexión generada por las dificultades metodológicas vinculadas a la erosión selectiva que afecta a las poblaciones de trabajadores por turnos. Estas dificultades han sido identificadas durante muchos años $\mathrm{y}$, sin embargo, han permanecido en gran medida sin ser abordadas hasta ahora. Justificada y prometedora a la vista de nuestros conocimientos y carencias, esta línea de análisis ha permanecido poco explotada. La situación actual parece favorable para su desarrollo; de hecho, el estudio de estas cuestiones está directamente vinculado a la problemática de los trabajos impulsados por A. Laville y S. Volkoff, que tienden a renovar la investigación sobre el envejecimiento en el trabajo y se interesa más ampliamente por todas las dimensiones de las condiciones de trabajo.

11 En el desarrollo de esta perspectiva, los marcos temporales del trabajo presentan características propias que deben ser tomadas en cuenta. Las exigencias temporales no sólo afectan al trabajador por turnos, sino también a su entorno familiar; por los tanto las disposiciones aplicadas no se limitan únicamente al trabajador por turnos. Por lo tanto, la evolución en el tiempo de la tolerancia a estas exigencias no puede considerarse simplemente como un envejecimiento biológico, sino que también debe relacionarse con los cambios en la célula familiar a lo largo de los años. El uso de horarios atípicos es el resultado de motivos individuales que forman parte de una historia personal y determinan el significado de las presiones que sufre, acepta, tolera o rechaza el trabajador. El compromiso con el trabajo por turnos y su continuidad o no es 
una variable de ajuste importante en el funcionamiento general del sistema personal de actividades (según la definición de Curie). Algunos cambios nacionales recientes en las condiciones institucionales y económicas (apertura del trabajo nocturno para las mujeres en todos los sectores productivos, reducción del trabajo nocturno a 35 horas en todos los hospitales, aumento de la práctica impuesta del trabajo a tiempo parcial) ofrecen oportunidades de investigación especialmente interesantes a este respecto.

\section{BIBLIOGRAFÍA}

Curie, C., \& Hajjar, V. (1987). Vie de travail, vie hors travail. La vie en temps partagé. In C. LevyLeboyer, \& J-C. Spérandio (Eds.), Traité de Psychologie du travail (pp. 37-55). Paris: PUF.

Gadbois, C., \& Queinnec, Y. (1984). Travail de nuit, rythmes circadiens et régulation des activités. Le Travail Humain, 47(3), 195-225.

Gadbois, C. (1990a). Dimensions temporelles de l'action et fiabilité des systèmes socio-techniques. In J. Leplat, \& G. de Terssac (Eds.), Les facteurs humains de la fiabilité dans les systèmes complexes (pp. 159-187). Toulouse: Octares.

Gadbois, C. (1990b). Travail posté et vie sociale: recherches actuelles et perspectives. Le Travail Humain, 53(2), 97-101.

Volkoff, S., Laville, A., \& Maillard, M. (1992). Ages et travail: Contraintes, sélection et difficultés chez les 40-50 ans. Travail et Emploi, 54, 20-33. 